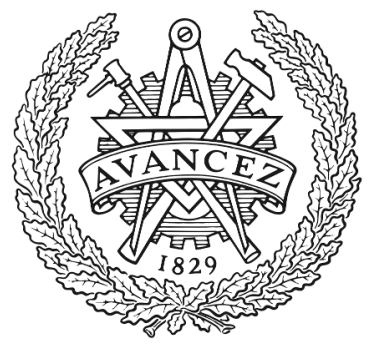

CHALMERS

UNIVERSITY OF TECHNOLOGY

\title{
Designing and characterizing MATE, the Chalmers mm-wave MIMO testbed
}

Downloaded from: https://research.chalmers.se, 2023-04-26 11:11 UTC

Citation for the original published paper (version of record):

Buisman, K., Eriksson, T. (2018). Designing and characterizing MATE, the Chalmers mm-wave

MIMO testbed. IET Conference Publications, 2018(CP741). http://dx.doi.org/10.1049/cp.2018.0747

N.B. When citing this work, cite the original published paper. 


\title{
Designing and characterizing MATE, the Chalmers mm-wave MIMO testbed
}

\author{
Koen Buisman, Thomas Eriksson \\ Department of Microtechnology and Nanoscience/ Department of Electrical Engineering, Chalmers University of Technology, \\ Gothenburg, Sweden, buisman@chalmers.se, thomase@chalmers.se
}

\begin{abstract}
In this paper, we report on the design and characterization of MATE, the mm-wave testbed at the Chalmers University of Technology. We elaborate around design choices of various parts of the testbed, baseband hardware, required software and $\mathrm{RF}$ frontend. We also perform a thorough characterization of the testbed, in terms of I/Q imbalance, stability, frequency offset, DC offsets, and ADC/DAC clock rate offset. The study shows that the utilized design techniques for baseband and RF frontend hardware are feasible, and also that many hardware imperfections are possible to accurately characterize, for subsequent calibration and digital compensation.
\end{abstract}

Index Terms-MIMO, distortion, measurement.

\section{INTRODUCTION}

Multi-antenna testbeds allow the study of limitations of future communications system on realistic hardware. Several testbeds in academia have been realized. For the example the Lund test-bed [1], which consist of 100 antennas with 40 $\mathrm{MHz}$ analog bandwidth each, operating at $3.7 \mathrm{GHz}$. ARGOS [2] at Rice University, which has 64 antennas, each with 20 $\mathrm{MHz}$ bandwidth, operating at either 2.4 or $5 \mathrm{GHz}$. NPL has realized a $2 \times 230 \mathrm{GHz}$ testbed [3] with $80 \mathrm{MHz}$ bandwidth.

Here we present our mm-wave testbed, MATE. Several requirements were imposed on the design, summarized here:

- Enables various kinds of mm-wave research.

- Large bandwidth.

- Off-the-shelf components.

- Independent transmitter and receiver.

- $\quad$ Easy to access by users.

- Possible to extend to real-time.

- Scalable for future requirements.

These requirements translated into a testbed that operates between 28 - $31 \mathrm{GHz}$, with $1 \mathrm{GHz}$ analog bandwidth per transmitter or receiver. MATE supports up to 18 channels, which can be used in various configurations, with up to 16 transmitters and up to 9 receivers. The results in this paper are based on the 8 transmitter (TX), single receiver (RX) configuration. Field-programmable gate arrays (FPGAs) are available at each individual transmitter or receiver. Between FPGAs there is sufficient date rate available to enable realtime operation. Furthermore, if more processing capabilities are needed, MATE can be extended with more FPGAs. The RF frontend is fully independent of the baseband hardware and software, thus can be replaced by other RF hardware, enabling different frequency bands.
MATE is easily accessible to users by a remote access interface, for which a MATLAB client has been created, which takes care of all communication aspects, enabling worldwide access. This client can be called as a function in MATLAB, thus allowing ease of integration in exiting software processing code. Design choices will be discussed in more detail in the following section.

\section{DESIGN OF THE TESTBED}

The mm-wave testbed can be dividend in three parts, baseband hardware, software and RF frontend hardware.

\section{A. Baseband hardware}

The MATE baseband transmitter and receiver hardware consists of analog-to-digital converters (ADCs), digital-toanalog converters (DACs), FPGAs, sample clocks and triggering. The baseband of the multi-antenna transmitter has its own sample clock, triggering and reference oscillator which is realized fully independent of the receiver, which has its own sample clock, triggering and reference oscillator.

To ensure the baseband signals are coherent, one sample clock is distributed to all DACs and another to all ADCs. Trigger signals are latched to the sampling clocks to enable coherent transmission or reception.

The baseband hardware (Fig. 1.) consists of National Instruments PXIe chassis (NI PXIe-1085), reference clock/trigger modules (NI PXIe-6674T), DACs (Active Technologies AT-1212), ADCs (NI-5771) and FPGAs (PXIe-7975R). One or two embedded controllers (NI PXIe$8880)$ control the system.

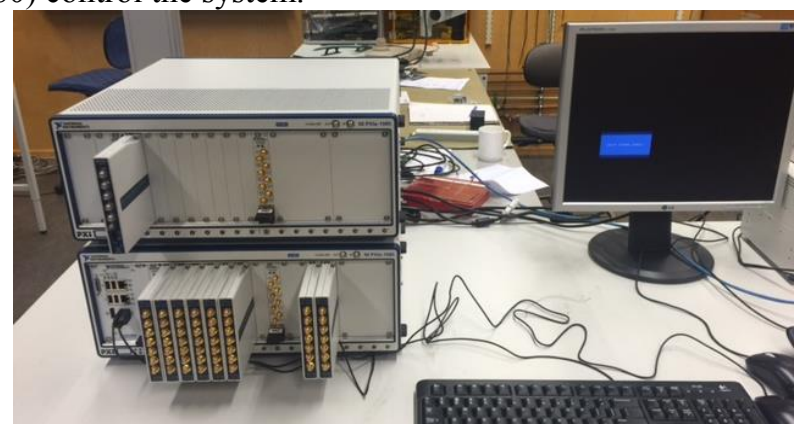

Fig. 1. MATE baseband hardware in $8 \mathrm{TX}, 1 \mathrm{RX}$ configuration, the bottom chassis hosts the FGPAs, DACs and clock/trigger module for the 8 channel multi-antenna transmitter. The top chassis hosts the FPGA, ADC and clock/trigger modulte for a single receiver. 


\section{B. Software}

The software takes care of synchronization, sample clocks, data transport between (host) memory and FGPA, as well as client-server communications. The software consists of several parts: code running on the FPGAs, code running on the host controller. Additionally the server running on the controller, as well as the webserver at a hosting provider and finally the client running at the user's computer.

The software for the FPGA and host controller that takes care of transmitting and receiving a signal in a coherent fashion is written in Labview. Firstly the system is initialized, then input data is written to the memory near the FPGAs, when a trigger is received this data is transmitted multiple times. A second trigger starts the acquisition at the receiver, where data from the $\mathrm{ADC}$ is transferred to the memory near the FGPA. Subsequently this data is transferred to the memory of the host controller, which passes the received data on to the server. The server is implemented by us in MATLAB and is similar to the server we constructed for our online RF WebLab [4] system. An overview is given in Fig. 2.

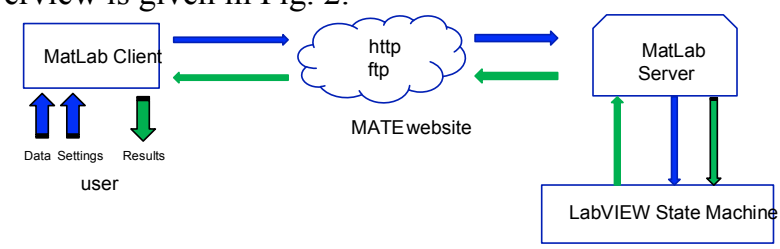

Fig. 2. Schematic overview remote access on-line interface.

\section{RF frontend hardware}

The mm-wave RF frontend is constructed using off-theshelf integrated circuits. A schematic is given in Fig. 3. The various components will be discussed subsequently.

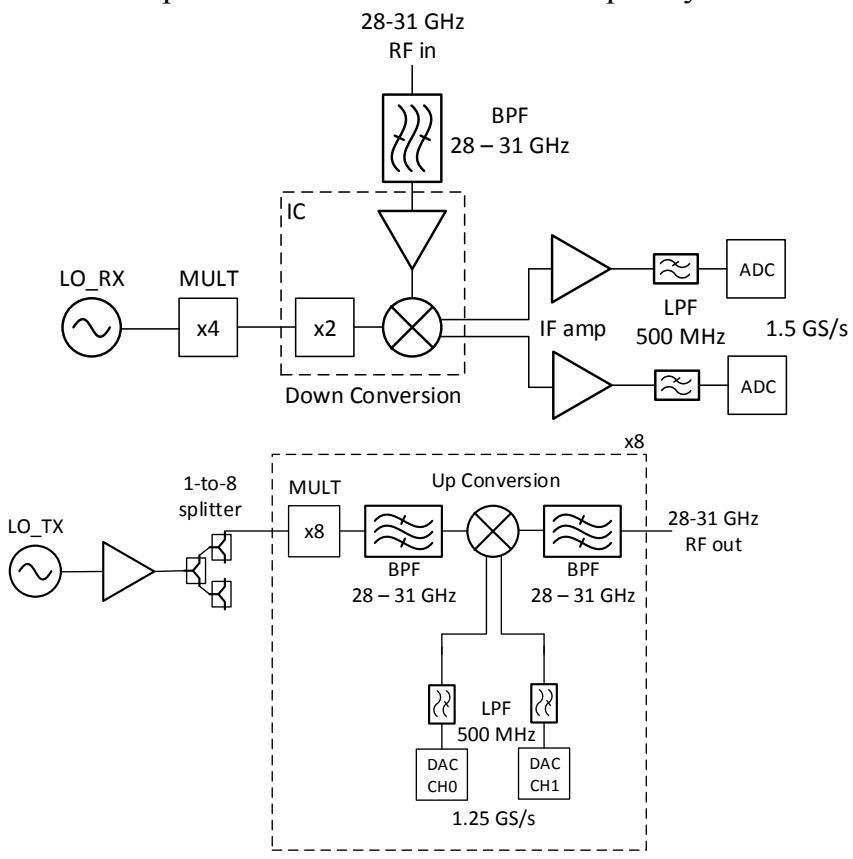

Fig. 3. Top: Schematic overview MATE receiver. Bottom: MATE transmitter, note only one of eight upconverting branches is shown.
The RF hardware is constructed from off-the-shelf components, supports $>1 \mathrm{GHz}$ analog bandwidth and operates between 28-31 GHz. To enable digital beamforming and MIMO signal processing, each TX chain of the system has to be coherent with respect to the other TX chains. To reach this goal we distribute a $\sim 3.5-3.875 \mathrm{GHz}$ local oscillator (LO) signal to each of the TX chains (Fig. 3.), where the LO is subsequently multiplied by a factor of 8 (Fig. 4. top). This choice was made to avoid the need to distribute $\sim 30 \mathrm{GHz}$ LO signals. An LO amplifier at $\sim 3.5$ $\mathrm{GHz}$ is used to compensate for the losses in distributing the LO. Note that if there is more than one RX, we employ a similar LO distribution for the RX as well. Unwanted products arising from the LO multipliers are suppressed with coupled stripline bandpass filters (Fig. 4. top), after which the LO signals drive IQ modulators (Fig. 4. top), where the baseband I and Q signals are provided by $1.25 \mathrm{GS} / \mathrm{s}$ DACs. After the IQ modulator a second identical bandpass filter is applied. The signal here can be connected to an antenna, as is the case in the configuration as discussed in this paper. To allow for larger distances, a power amplifier can be inserted.

The RX chain (Fig. 3.) is similar to the TX chains, one exception is that we use a direct IQ downconverter IC (Fig. 4. bottom) with integrated $\times 2$ LO multiplier. Thus the LO multiplier we have constructed is $\mathrm{x} 4$. The downconvertor IC contains a low noise amplifier and gives out the downconverted I and Q, which are subsequently amplified, filtered and digitized by $1.5 \mathrm{GS} / \mathrm{s}$ ADCs.

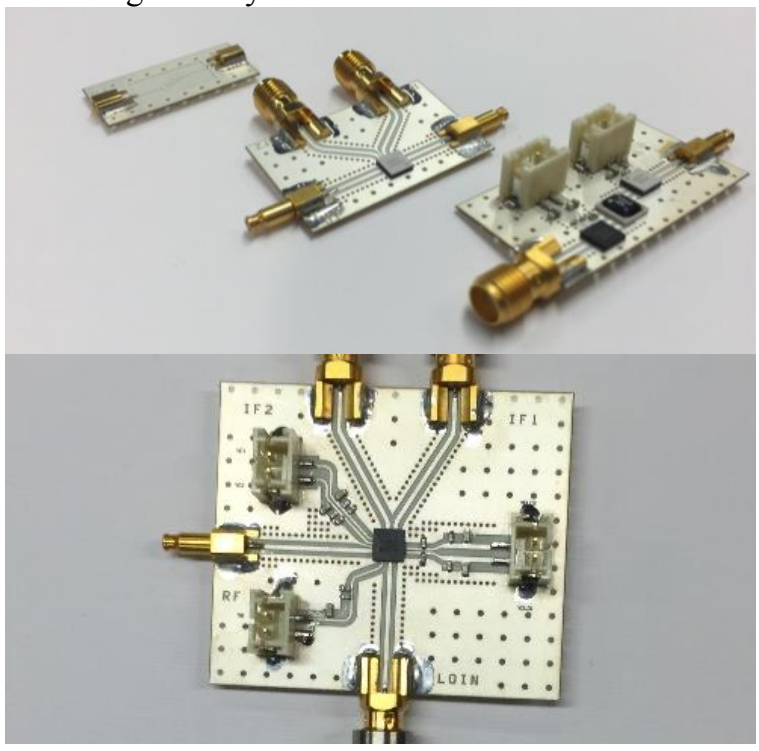

Fig. 4. Top: MATE realized circuit boards, Top: 28-31 GHz bandpass filter, TX IQ upconvertor and TX x8 multiplier. Bottom: MATE RX IQ downconvertor.

The full system is shown in Fig. 5. On the left the multiantenna transmitter can be seen, on the right a single receiver. Note that the path over which we transmit is about $10-20 \mathrm{~cm}$. For many (DSP) experiments this short path suffices. In the future we will also perform experiments with power amplifiers at each TX chain. Patch antennas are used and are shown in Fig. 6. 


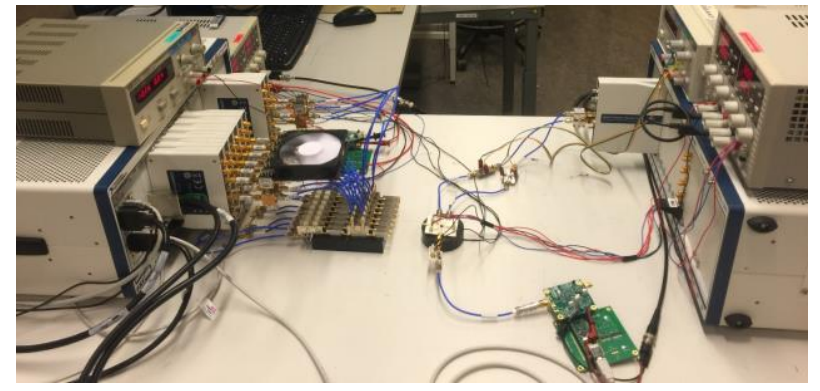

Fig. 5. The MATE MIMO testbed in the $8 \mathrm{TX}, 1 \mathrm{RX}$ configuration, on the left the multi-antenna transmitter and the right a single receiver.

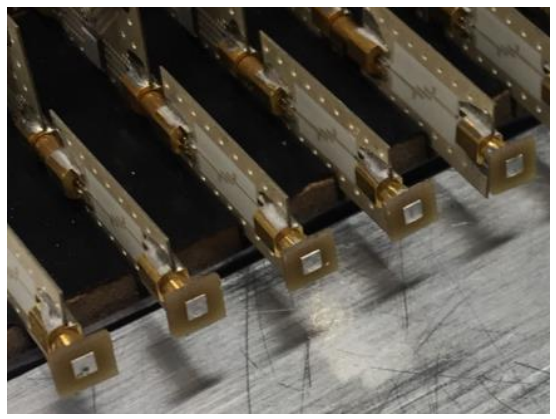

Fig. 6. Installed patch antennas at the multi-antenna transmitter.

TABLE I. KEY FEATURE OF THE MATE TESTBED

\begin{tabular}{|l|c|}
\hline & MATE \\
\hline Operating frequency & $28-31 \mathrm{GHz}$ \\
\hline Analog Bandwidth per TX/RX chain & $1 \mathrm{GHz}$ \\
\hline No. TX & 8 (extendable to 16) \\
\hline No. RX & 1 (extendable to 9) \\
\hline No. FPGAs & 18 \\
\hline RF Output power per TX chain & Max. -4 dBm \\
\hline Noise figure RX & $3 \mathrm{~dB}^{\mathrm{a}}$ \\
\hline
\end{tabular}

The construction of the hardware and making the system operational was completed in December 2016. By October 2017 over 10.000 measurements have been performed by us and collaborators. Some key features of the testbed are summarized in Table 1. When comparing our testbed with existing testbeds in academia [1-3], testbeds realized in the low $\mathrm{GHz}$ region have much lower analog bandwidth, but more channels. We have realized a large analog bandwidth as well as a high channel count for mm-wave testbeds.

\section{CHARACTERIZATION OF THE TESTBED}

In the mm-wave testbed, there are many different kinds of hardware imperfections that affect the quality of the received signals. Here, we study these imperfections one by one, sometime through innovative techniques to isolate the phenomenon, other times through straightforward frequencyor time-domain techniques. This identification of the various imperfections is a prerequisite to calibration of the array and compensation of the artefacts. All experiments were performed at a center frequency of $28.5 \mathrm{GHz}$.

\section{A. First experiment}

In a first experiment, we transmit a signal with a bandwidth of $100 \mathrm{MHz}$, offset by $150 \mathrm{MHz}$ from the center frequency, only using transmit antenna 7 . The spectrum of the received signal is illustrated in Fig. 7.
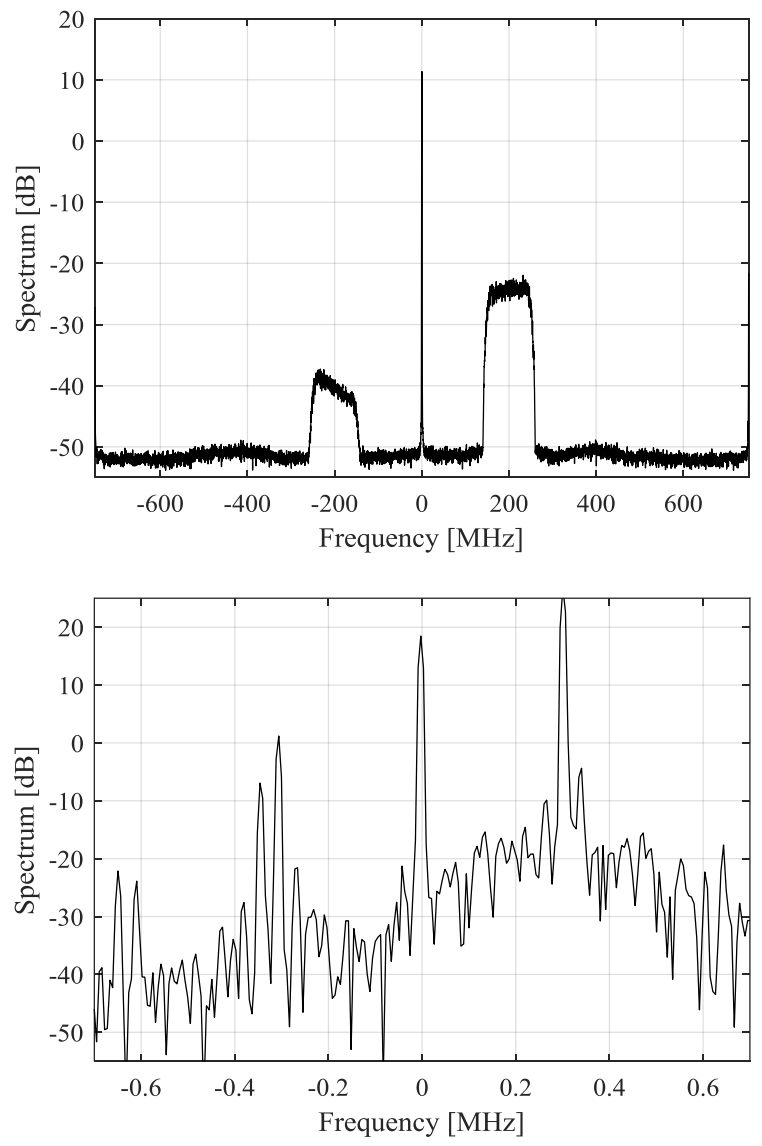

Fig. 7. Top: Spectrum of a transmitted signal at $400 \mathrm{MHz}$ offset, with a bandwidth of $100 \mathrm{MHz}$. Bottom: We see a zoomed-in spectrum, centered around $\mathrm{DC}(0 \mathrm{~Hz})$. LO leakage at transmitter and receiver are revealed.

Already in this simple transmission, many issues are visible. First, we see that the signal shows some frequency selectivity within its band at $200 \mathrm{MHz}$. We also clearly see a signal at the mirror frequency of $-200 \mathrm{MHz}$, which is due to (also frequency selective) I/Q imbalance (IQI) in either TX or RX. At around $0 \mathrm{~Hz}$, we see a strong signal indicating a DC offset. Zooming in, we can see a strong peak at exactly 0 $\mathrm{Hz}$, which is coming from a LO leakage in RX, leading to a DC offset. Another strong signal is at around $300 \mathrm{kHz}$, which indicates two things; there is a LO leakage at TX, and we have a frequency offset of around $300 \mathrm{kHz}$. The mirror at $300 \mathrm{kHz}$, due to I/Q imbalance, is also seen. The RX LO leakage ends up at around DC since the RX LO is mixed with itself, ending up at $0 \mathrm{~Hz}$, and the TX LO leakage is 
mixed with the RX LO, ending up at the frequency offset between TX and RX.

In the following, we focus on one issue at the time, trying to understand exactly the characteristics of the testbed.

\section{B. Frequency selectivity and $I / Q$ imbalance}

By transmitting a sequence of overlapping spectra, we can in Fig. 8 study the frequency selectivity of the testbed, both for the intended band and the mirror frequency band (due to IQI).
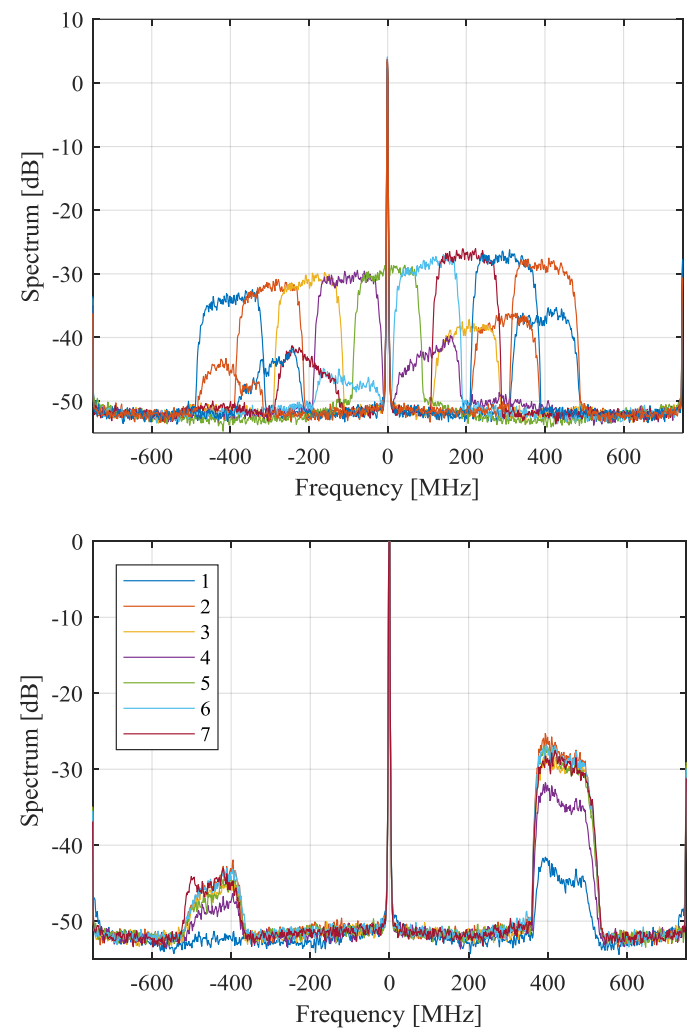

Fig. 8. Top: A sequence of overlapping spectra. The transmitted signal band is stronger than the mirror band in all transmissions. Bottom: Transmission at around $450 \mathrm{MHz}$, using all different antennas 1-7. Antenna 1 has a weaker signal than the rest.

There is some clear frequency selectivity, with about 5 $\mathrm{dB}$ differences over the band. The IQI shows an even stronger selectivity. In the bottom part of Fig. 8, we have transmitted the same signal over the $7 \mathrm{TX}$ antennas one by one. Also here we see some power variation, in particular antenna 1 is weaker than the others. All antennas seem to have approximately the same amount of IQI.

\section{Frequency offset}

There are two kinds of frequency offsets in the testbed, one in the TX and RX LOs and one in the ADC/DAC clocks. Both leads to frequency shifts in the received signal, but they have different characteristics. A difference in the LO frequencies leads to a shift of the entire spectrum, while a difference in DAC and ADC clock rates leads to an expansion or compression of the spectrum.
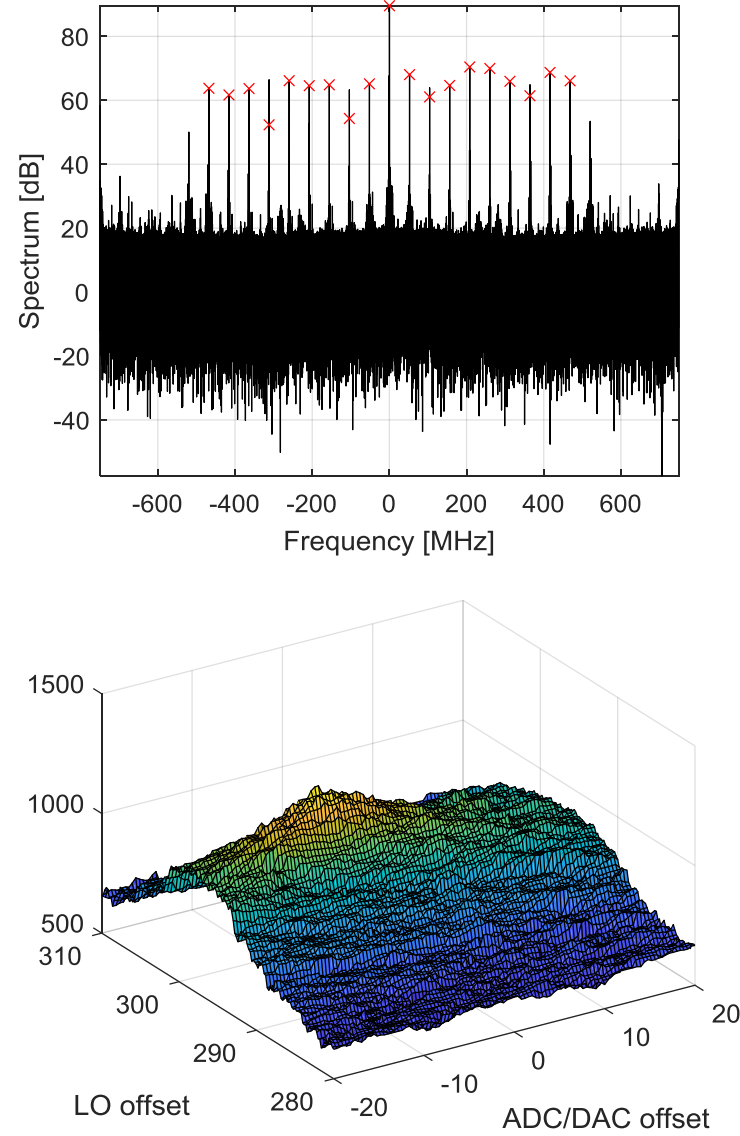

Fig. 9. Top: A peaky received spectrum when uniformly spaced sinusoids are transmitted. The red crosses hitting the peaks indicates that we have found good ADC/DAC and LO offsets. Bottom: A parameter sweep reveals the correct frequency offsets.

To find the two offsets, we transmit a signal that is a sum of sinusoids spaced $50 \mathrm{MHz}$ apart. Then, we adjust the compression/expansion and the offset of the received spectrum until our received frequency grid optimally matches the transmitted one. Fig. 9 illustrates the concept, with the small red crosses indicating the TX grid, which is translated and expanded/compressed until it optimally hits the spectral peaks of the received signal. The bottom part of the figure illustrates a parameter sweep over the two offsets, revealing that the testbed has an $\mathrm{LO}$ offset of $290 \mathrm{kHz}$, and a DAC/ADC offset of $-6 \mathrm{kHz}$. The DAC/ADC offset is very stable, with variations over long time in the order of $100 \mathrm{~Hz}$, while the LO offset can vary $+/-10 \mathrm{kHz}$ when measured over a longer time period (days or weeks).

\section{Beamforming}

When the transmitted signals over different antennas are phase-shifted such that their phases align at the receiver, the received signal is considerably stronger than with no phase alignment. This procedure is called beamforming, and is the topic of this subsection.

In this study, we transmit a reference sinusoid at antenna 7, and a phase-shifted sinusoid with the same frequency on the other antennas, one by one. When the signals are phase- 
aligned at the receiver, we will see a power maximum. In Fig. 10, we plot the received power level while the phase shift is swept over the interval for each antenna.

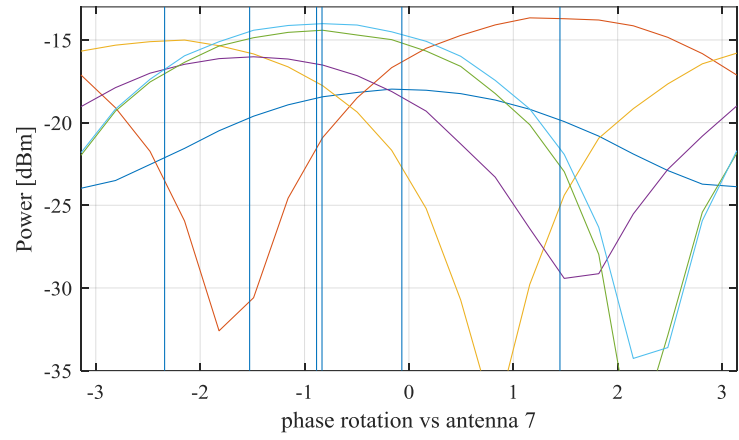

Fig. 10. Plot of the received power when transmitting sinusoids on two antennas (antenna 7 as reference) and sweeping the phase difference.

This study can be used to understand how the signal on each antenna should be phase-shifted to provide phase coherence with antenna 7 , thereby enabling phase coherency of the entire array. The vertical lines in the figure indicates, for each antenna, its phase shift to align with antenna 7.

\section{E. Stability analysis}

Stability is an important concept in a testbed. For parameters that are stable, we will be able to apply calibration to mitigate problems, while for unstable parameters adaptive techniques will be needed. To study the stability of the testbed, we perform multiple experiments and verify their consistency.

In a first set of experiments, we repeatedly transmit a sinusoidal waveform at $100 \mathrm{MHz}$ offset, and study the power of the received signal. In Fig. 11, we see the power stability of 8 consecutive transmissions for each of the 7 transmitters. Channel 1 leads to a lower received power than the others, but the stability in each case is very good, better than $0.1 \mathrm{~dB}$ variation between the transmissions.

In the bottom figure, we see 5 transmissions over channel 6. Again we see a very good amplitude stability, but here we can see some frequency offset variations; those are as expected, and entirely within the limits of the quality of the local oscillators.

\section{CONCLUSION}

We have demonstrated that the MATE testbed at Chalmers is very suitable as a tool for future mm-wave $5 \mathrm{G}$ communication research. By elaborating around design choices, and performing a sequence of detailed experiments, we show that the testbed is a stable and capable tool for making experiments, and the remote access interface makes studies extremely convenient. The capability to create a phase-aligned signal paves the wave for MIMO and massive MIMO studies focused on $5 \mathrm{G}$ communications, but also for studies in other disciplines, such as medical technology or radar studies.
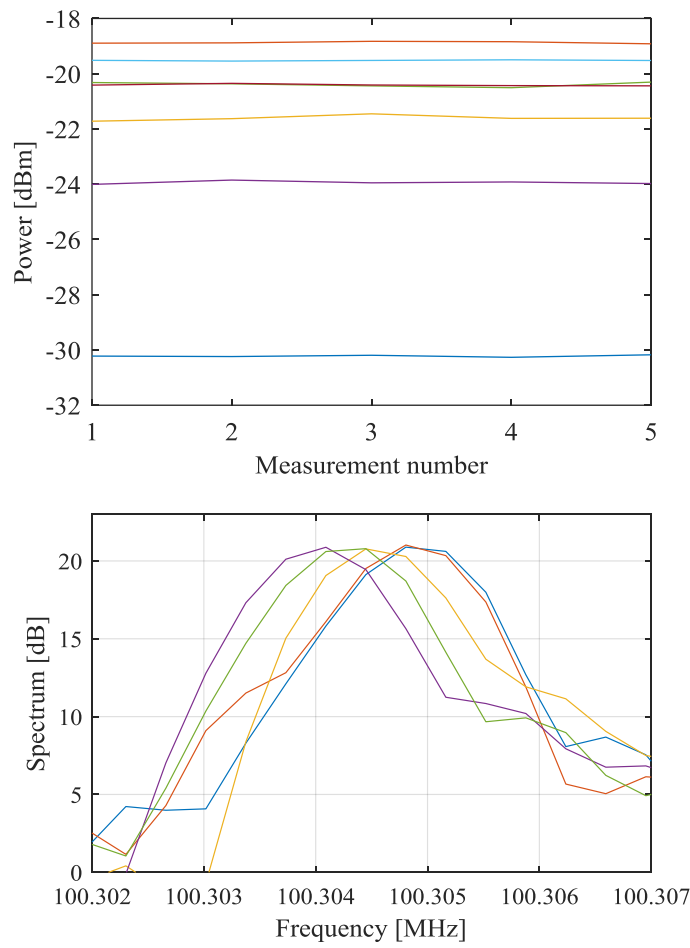

Fig. 11. Study of the stability over multiple transmissions. The power is very stable, while the frequency offset shows some variability between transmissions $(\sim 1 \mathrm{kHz})$.

\section{ACKNOWLEDGMENT}

The results in this paper are partly the result of the project MET5G Metrology for 5G communications. This project has received funding from the EMPIR programme co-financed by the Participating States and from the European Union's Horizon 2020 research and innovation programme. Furthermore this research has been carried out in the GigaHertz centre in a joint research project financed by Swedish Governmental Agency of Innovation Systems (VINNOVA), Chalmers University of Technology, Ericsson, Infineon Technologies Austria, Ampleon, SAAB, QAMCOM, and National Instruments. The work has also been financed by the strategic innovation program "Smartare Elektroniksystem", a common effort by VINNOVA, Formas and Energimyndigheten.

The authors would like to thank Vasileios Tokmakis for his contributions to creating the MATE testbed.

\section{REFERENCES}

[1] J. Vieira et al., "A flexible 100-antenna testbed for Massive MIMO," 2014 IEEE Globecom Workshops (GC Wkshps), Austin, TX, 2014, pp. 287-293.

[2] C. Shepard, H. Yu, N. Anand, E. Li, T. Marzetta, R. Yang, and L. Zhong, "Argos: Practical many-antenna base stations," in Proc. ACM MobiCom, 2012.

[3] T. H. Loh, D. Cheadle and P. Miller, "A millimeter wave MIMO testbed for 5G communications," 2017 89th ARFTG Microwave Measurement Conference (ARFTG), Honololu, HI, 2017, pp. 1-4.

[4] RF WebLab at Chalmers - dpdcompetition.com [online] accessed October 27, 2017. 